\title{
Table of cases - Tabla de jurisprudencia
}

\author{
AUSTRALIA \\ Armacel Pty Ltd v Smurfit Stone Container Corp [2007] FCA 1928
} 374

\section{ENGLAND (UNITED KINGDOM)}

ABCI (formerly Arab Bus Consortium Intl Fin and Invest Co v Banque Franco-Tunisiennee) [2002]

1 Lloyd's Rep 511 374

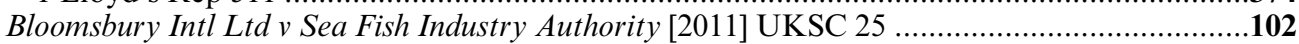

Boyd v The Army Prosecuting Authority [2002] UKHL 31 ...................................................149

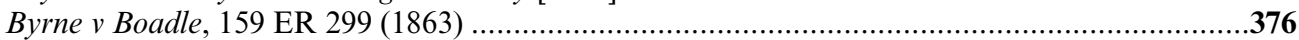

Cambridge Water Co Ltd v Eastern Counties Leather plc [1994] 2 AC 264 ...............................278

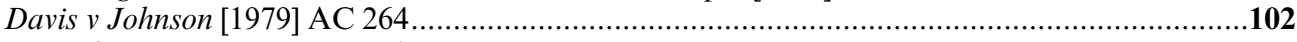

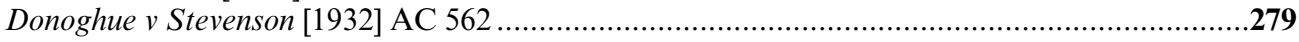

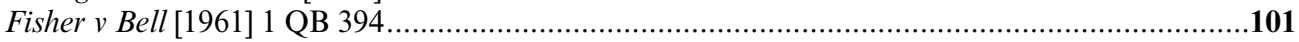

Grey v Pearson 10 ER 1216, (1857) 6 HL Cas 61............................................................101

Heydon's Case (1584) 3 Co Rep 7a ...........................................................................101

Inco Europe Ltd v First Choice Distribution [2000] UKHL 15 .............................................101

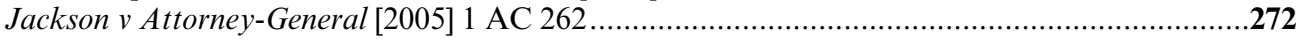

Kleinwort Benson Ltd v Lincoln City Council [1999] 2 AC 349...............................................161

Litster v Forth Dry Dock \& Engineering Co Ltd [1990] 1 AC 546 .............................................101

London and North Eastern v Berriman [1946] AC 278 ...........................................................101

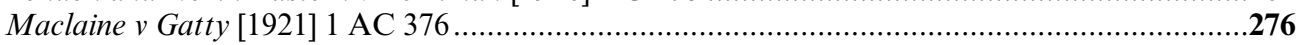

McCartan Turkington Breen v Times Newspapers Ltd [2001] 2 AC 277 ..................................102

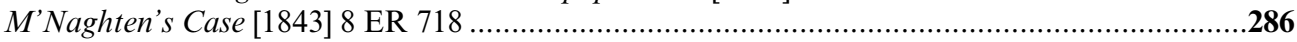

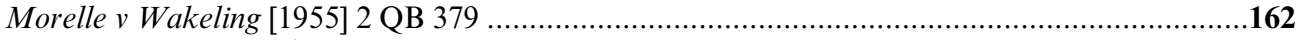

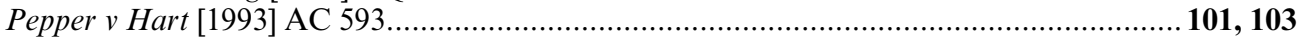

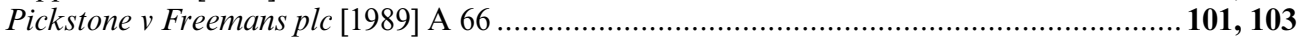

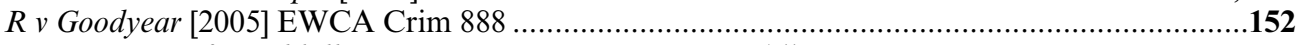

$R v$ Governor of Brockhill Prison, ex parte Evans [2000] 4 All ER 15 .....................................277

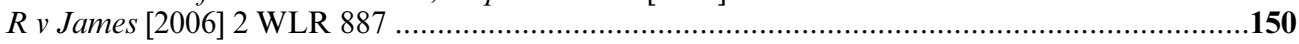

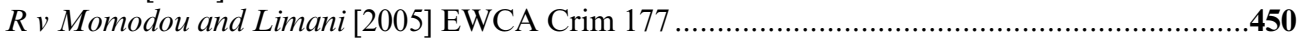

$R v$ Secretary of State for Transport, ex parte Factortame Ltd [1991] 1 AC 603 ...................91, 121

$R v$ Secretary of State for the Environment, Transport and the Regions, ex parte Spath Holme Ltd

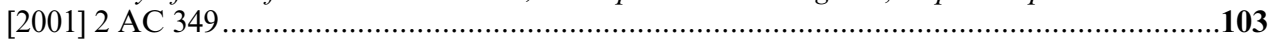

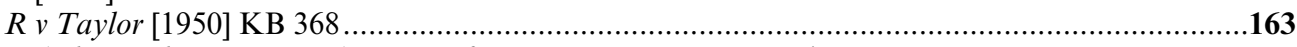

$R$ (Electoral Commission) v City of Westminster Magistrates' Court [2010] UKSC 40 ..............101

$R$ (on the application of Quintavalle) $v$ Secretary of State for Health [2003] UKHL 13..............102

Rylands v Fletcher [1868] LR 3 HL 330 ..............................................................................278

Thoburn v Sunderland City Council [2002] EWHC 195 (Admin) ......................................90, 271

Thomas $v$ National Union of Mineworkers (South Wales Area) [1985] 2 All ER 1.....................277

Trident Turboprop (Dublin) Ltd v First Flight Couriers Ltd [2009] EWCA Civ 290 ....... 171-6, 639

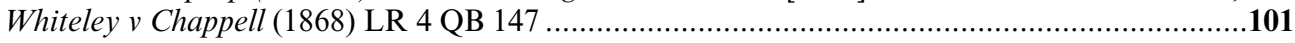

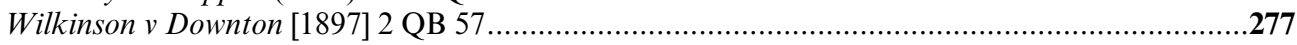

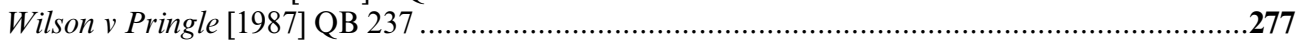

Young v Bristol Aeroplane Co Ltd [1944] KB 718 ................................................................162 


\section{EUROPEAN COURT OF HUMAN RIGHTS}

Morris v United Kingdom, (2002) 34 EHRR 52

\section{EUROPEAN UNION}

Case C-550/07 Akzo Nobel Ltd v European Commission [2010] ECR I-8301 $.23,36$

Case 26/62, NV Algemene Transport- en Expeditie Onderneming van Gend and Loos v Netherlands

Inland Revenue Administration, [1963] ECR 1, [1963] CMLR 105

$105,304,355$

Dictamen TJ 2/91, de 14 de diciembre de 1991

Dictamen TJ $1 / 2003$, de 7 de febrero de 2006

Dictamen TJ 1/13, de 14 de octubre de 2014

STJ de 5 de febrero de 1962, As 26/62, Van Gend en Loos......

STJ de 5 de febrero de 1963, As 26/62, NV Algemene Transport- en Expeditie Onderneming van

Gend \& Loos $v$ Netherlands Inland Revenue Administration ..... $105,130,304,355$

STJ de 31 de marzo de 1971, As 22/70, Comisión v Consejo o AETR 356

STJ de 14 de octubre de 1976, As 29/76, LTU Lufttransportunternehmen v Eurocontrol ................126

STJ de 27 de septiembre de 1988, As 81/87, Daily Mail ...................................................................348

STJ de 4 de mayo de 1995, As 7/94, Landesamt für Ausbildungsförderun Nordhein-Westfalen....339

STJ de 9 de marzo de 1999, As C-212/97, Centros....................................................................348

STJ de 8 de junio de 1999, As C-337/97, Meeusen .....................................................................339

STJ de 11 de julio de 2002, As 60/00, Carpenter ...................................................................339

STJ de 5 de noviembre de 2002, As C-208/00, Überseering .....................................................348

STJ de 17 de septiembre de 2002, As 413/99, Baumbast y R.....................................................339

STJ de 23 de septiembre de 2003, As C 109/01, Akrich ...........................................................339

STJ de 30 de septiembre de 2003, As C-167/01, Inspire Art ....................................................348

STJ de 19 de octubre de 2004, As 200/02, Zhu y Chen ..............................................................339

STJ de 13 de septiembre de 2005, As C-176/03, Comisión v Consejo...........................................331

STJ de 16 de diciembre de 2008, As C-210/06, Cartesio ..............................................................348

STJ de 23 de febrero de 2010, As 310/08 y 480/08, Ibrahim y Teixeira .........................................339

STJ de 14 de septiembre de 2010, As C-550/07, Akzo Nobel Ltd v Comisión Europea .............23, 36

STJ de 8 de marzo de 2011, As C 34/09, Ruiz Zambrano...............................................................340

STJ de 5 de mayo de 2011, As 434/09, Mc Carthy..................................................................340

STJ de 15 de noviembre de 2011, As C-256/11, Dereci y otros ...................................................340

\section{$\mathrm{MEXICO}^{1}$}

Pleno de la SCJN el expediente 912/2010 concerniente al cumplimiento de la sentencia de la Corte Interamericana de Derechos Humanos recaída en el caso Radilla Pacheco SCJN, Amparo en revisión 1475/98, Semanario Judicial de la Federación y su Gaceta, Tomo XI, Marzo de 2000, 442

SCJN, Pleno, Contradicción de Tesis 293/2011, Gaceta del Semanario Judicial de la Federación,

Libro 5, Abril de 2014, Tomo I, 96. 211-13

Segundo Tribunal Colegiado en materia civil del tercer circuito, Amparo directo 227/2009, Semanario Judicial de la Federación.

1 Las decisiones emitidas por la Suprema Corte de Justicia de la Nación son accesibles en $<$ www2.scjn.gob.mx/>. Las decisiones de otros órganos pueden localizarse en $<$ http://sjf.scjn.gob. $\mathrm{mx} / \mathrm{SJFSem} /$ Paginas/SemanarioIndex.aspx $>$. 


\section{NEW ZEALAND}

Paper Reclaim Ltd v Aotearoa Intl Ltd [2006] 3 NZLR 188

\section{SPAIN $^{2}$}

ATC 191/2004, de 26 de mayo (Tribunal Constitucional) .....................................................203

ATC 201/2004, de 27 de mayo (Tribunal Constitucional) ................................................203

STC 5/81, de 13 de febrero (BOE 24.2.1981) ...........................................................................128

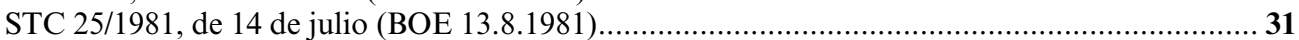

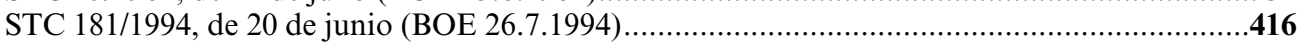

STC 108/2003, de 2 de junio (BOE 1.7.2003) ….......................................................................203

STC 46/2004, de 23 de marzo (BOE 23.4.2004) ..............................................................203

STC 150/2004, de 20 de septiembre (BOE 22.10.2004) .........................................................203

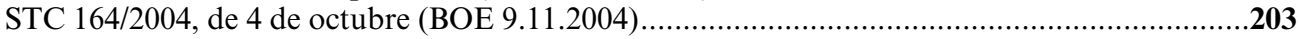

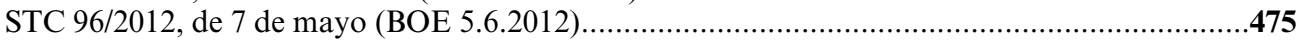

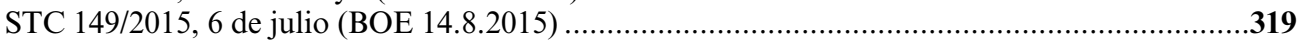

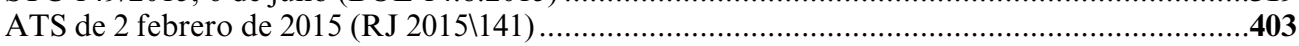

STS de 20 de noviembre de 1989 (RJ 198918206) .......................................................................559

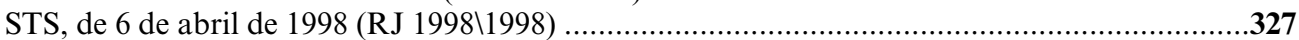

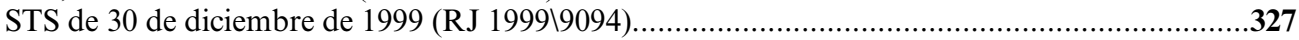

STS de 28 de febrero de 2007 (RJ 2007\4846)...............................................................559

STS de 16 de diciembre de 2009 (RJ 702/2010) (CENDOJ 8466/2009) .....................................210

STS de 29 de julio de 2011 (RJ 2011 16285$)$.............................................................................330

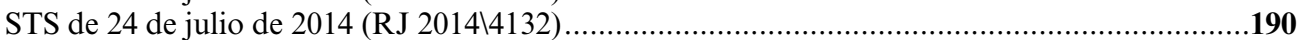

STS de 20 de octubre de 2014 (CENDOJ 28079110012014100504) .......................................213

STSJ Cantabria de 31 de julio de 1997 (AS 1997\2426) ........................................................559

STSJ Santa Cruz de Tenerife de 16 de noviembre de 2001 (JUR 2002\42170)..........................559

\section{UNITED STATES}

Asahi Metal Indus Co v Superior Court, 480 US 102 (1987)....................................................147

Asante Techs, Inc v PMC-Sierra, Inc 164 FSupp2d 1142 (ND Cal 2001) ..................................303

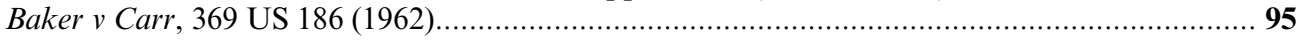

Bob Jones University v United States, 461 US 574 (1983) ....................................................103

Boston Stock Exchange v State Tax Commission, 429 US 318 (1977) ......................................145

Burger King Corp v Rudzewicz, 471 US 462 (1985).......................................................147

Chevron Corp v Donziger, No 11 Civ 0691 (LAK), 2013 WL 5548913 (SDNY October 7, 2013)

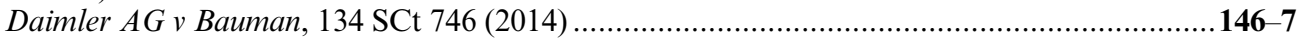

De Canas v Bica, 424 US 351 (1976) ............................................................................28

Director, Office of Workers' Compensation Programs, United States Dept of Labor v Perini North

River Assoc, 459 US 297 (1983) .......................................................................................161

Doe v Roman Catholic Diocese of Galveston-Houston, 408 FSupp2d 272 (SD Tex 2005).............305

Durham v United States, 214 F2d 862 (DC Cir 1954) ............................................................286

Erie Railroad Co v Tompkins, 304 US 64 (1938)............................................................148, 370

2 La legislación española, incluida la producida por las comunidades autónomas, y las sentencias del Tribunal Constitucional se publican en el BOE $<$ http://boe.es/ $>$. Existen diversas bases de jurisprudencia como la gratuita y gestionada por el Poder Judicial español CENDOJ < http://www. poderjudicial.es/search/indexAN.jsp>. 


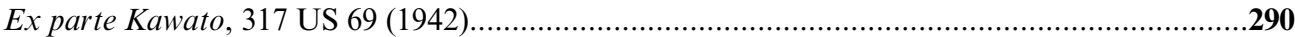

First National City Bank v Banco Nacional de Cuba, 406 US 759 (1972) ...................................306

Florida v Jardines, 133 SCt 1409 (2013)........................................................169-71, 176, 639

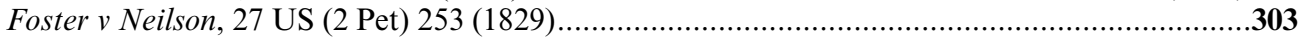

Gall v Topcall Intl, AG, No Civ A 04-CV-432, 2005 WL 664502 (ED Pa 2005).........................147

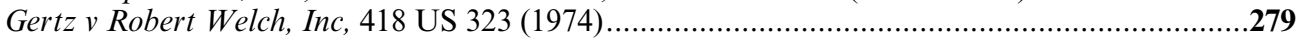

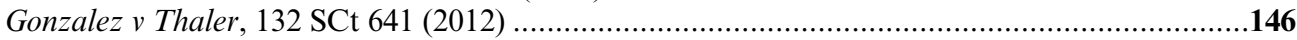

Hart v Massanari, 266 F3d 1155 (9th Cir 2001) ..........................................................44, 68

Helicopteros Nacionales de Colombia, SA v Hall, 466 US 408 (1984) .....................................147

Hickman v Taylor 329 US 495 (1947) ................................................................22, 60, 86

International Shoe Co $v$ Washington, 326 US 310 (1945) .......................................146, 147, 370

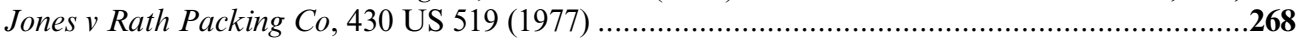

Jones $v$ United States, 529 US 848 (2000) ..............................................................................104

Klein v Freedom Strategic Partners, LLC, 595 FSupp2d 1152 (D Nev 2009)...........................445

Marbury v Madison, 5 US (1 Cranch) 137 (1803) .................................................92, 99, 144

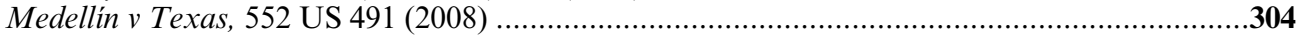

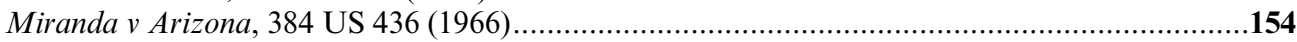

Morrison v Natl Austl Bank Ltd, 130 SCt 2869 (2010).....................................................147-8

Mossman v Higginson, 4 US (4 Dall) 12 (1800) .....................................................................147

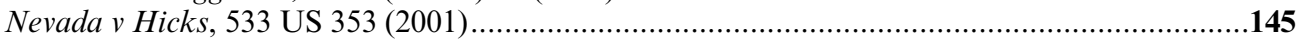

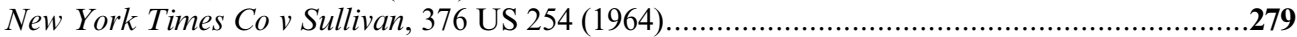

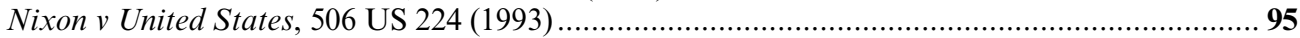

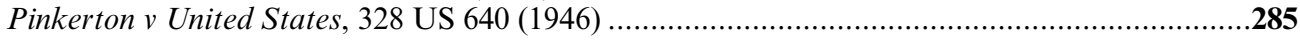

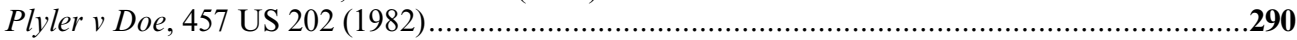

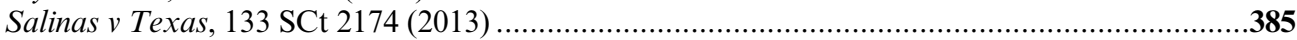

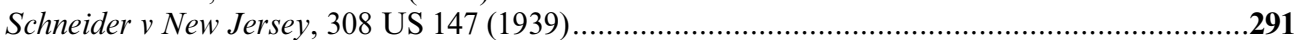

Shelby County v Holder, 133 S Ct 2612 (2013) ........................................................................ 92

Société Nationale Industrielle Aérospatiale v US District Court for the Southern District of Iowa,

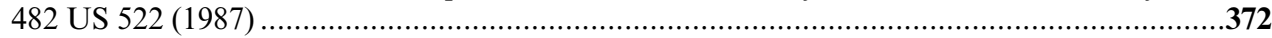

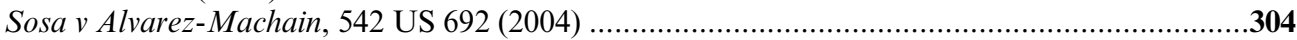

Southern Pacific Co v Jensen, 244 US 205 (1917) ................................................................161

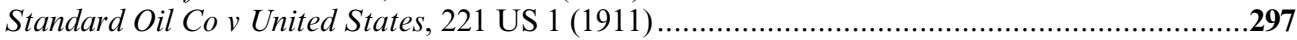

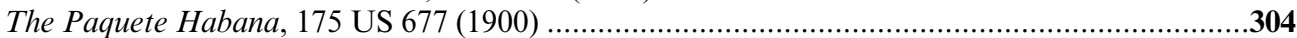

Trammel v United States, 445 US 40 (1980) .....................................................5, 83, 373

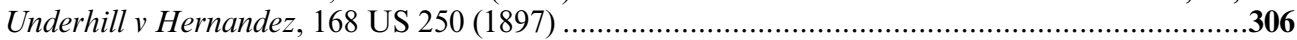

United States v Bruguier, 735 F3d 754 (8th Cir 2013) .............................................................99

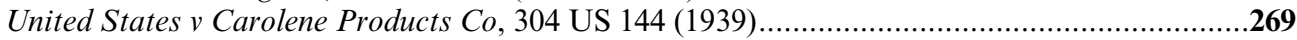

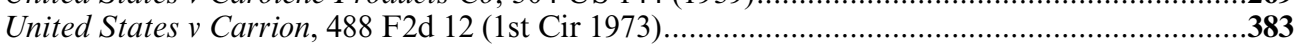

United States v Deutsches Kalisyndikat Gesellschaft, 31 F2d 199 (SDNY 1929)...........................305

United States v Si, 333 F3d 1041 (9th Cir 2003) ......................................................................289

Volkswagenwerk Aktiengesellschaft v Schlunk, 486 US 694 (1988) .........................................372

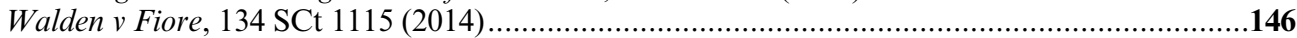

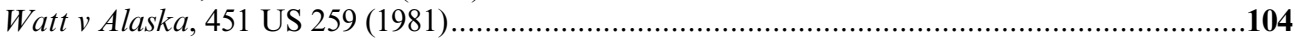

World-Wide Volkswagen Corp v Woodson, 444 US 286 (1980) ...........................................147 